\title{
THE EFFECT OF MAGNETIC STIMULATION ON POTENTIAL RHYTHM OF CEREBRAL CORTEX
}

\author{
Y.Hu* ${ }^{\dagger}$, YM.Feng*, MS.Wang*, WW.Lu ${ }^{\dagger}$ \\ *Department of Biomedical Engineering, Tianjin University, Tianjin, 300072,P.R.C \\ ${ }^{\dagger}$ Department of Orthopaedic Surgery, University of Hong Kong, Hong Kong, P.R.C \\ E-mail: yhud@hkusua.hku.hk
}

\begin{abstract}
An approach using magnetic stimulation to modulate the electromagnetic potential rhythm of the cerebral cortex to induce sleep is proposed in this paper. Animal experiments were designed and carried out to examine this approach. The results showed that, in comparison with a control group, magnetic stimulation can influence and modulate the activities of brain potentials, and consequently promote the efficiency of the sleep process $(p<0.01)$.

Key words: magnetic stimulation, pulsed magnetic field, potential rhythm of cerebral cortex
\end{abstract}

\section{Introduction}

The interaction between electroencephalographic(EEG) activity and external physical factors has become a research area of much interest to many investigators. Recently, many studies have been carried out to examine the effect of magnetic stimulation on the nervous system and EEG activity. The technique of transcutaneous magnetic stimulation has been established and is relatively advanced in comparison with electrical stimulation techniques ${ }^{[1-3]}$. Most of the work carried out to date has focused on neurological stimulation using pulsed magnetic fields. Some reports proposed the initial on the effective mechanism in every aspect of nervous system and EEG activities stimulated by electric and magnetic action. In this study, we propose a new method to simulate the sleep process using an EEG pattern. This magnetic field stimulation forms an induced current in the brain, stimulating different sides of the brain, and in particular in the region of the Hess area. The magnetic stimulation causes an impulse and a proliferation of reactions from tipsier cells, which actively transmit messages to the neural network. The active sleep center can then be triggered and will begin to resonate in synchronization.

\section{Materials and Method}

Four cats ( 2 males and 2 females) were used in this study, weighing $2.8,3.0,3.1$ and $3.3 \mathrm{Kg}$ respectively. The cats for the experiments were placed electrodes in the brain of each cat by surgery. In order to exclude interference from external electromagnetic signals and also to minimize the influence of the operation on the deposited electrode and setting coils, the cats were put into a screened room for two weeks post-operatively. A good level of air flow and light transmission in the room was maintained throughout.

The magnetic coil was mounted on the cat's skull over the thalamic rein for all the experiments conducted during this study, and the EEG, eletrooculogram (EOG) and electromyogram (EMG) were monitored and recorded. Then the Rontschaffch range estimation method was used for estimating the cat's sleeping condition.

The duration from the beginning of experiment to the first appearance of slow wave sleep was selected as the main parameter for evaluation in this study.

The magnetic stimulating device was designed by the Dept. of BME of Tianjin Univ. to generate simulated EEG waves by means of a variable magnetic field. A block diagram of the device is shown in Fig.1. The device consisted of 2 parts: the stimulation coil and the circuit to generate pulsed magnetic field, including charging circuit, energy storage condenser, high voltage and large current switch.

The voltage across the capacitor rises to a predetermined value under the charge of the high voltage circuit. The high voltage and large current switch was controlled by the microcomputer system which was opened and closed at a determined frequency to make the charges

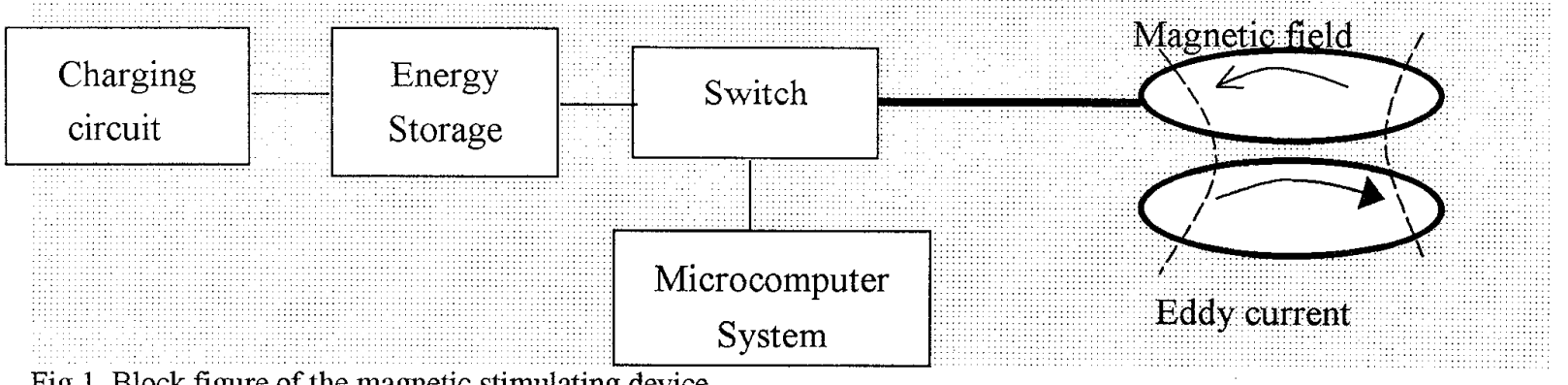

Fig. 1. Block figure of the magnetic stimulating device 
stored in energy storage condenser discharge to the coil. As such, a pulsed field was formed.(Fig.2)

In this figure, the ascending time $\left(\mathrm{t}_{\mathrm{m}}\right)$ was $0.1 \mathrm{~ms}$, the ratio of ascending time to descending time was about 1:5, and the largest magnetic induction intensity at the center point of coil $\left(B_{\mathrm{m}}\right)$ was $5.0 \times 10^{-3} \mathrm{~T}$. The coil diameter was $7 \mathrm{~cm}$, wired in 40 turns. The frequency of the pulsed magnetic field was controlled by the microcomputer control system. This device allowed for selection of 4 different pulse frequencies: $9 \mathrm{~Hz}$, $8 \mathrm{~Hz}, 6 \mathrm{~Hz}$ and $4 \mathrm{~Hz}$. In addition, there were also 4 different durations of magnetic stimulation could be applied.

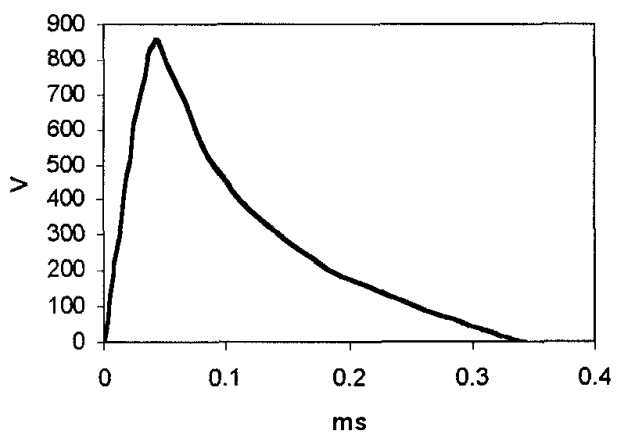

Fig. 2 The waveform of pulsed magnetic field

While the cats were conscious, a simulated brain wave pattern of slow wave sleep was coupled from the coil to cat's brain, in order to induce the encephalic electrical activity to synchronize gradually with the external low-frequency magnetic fields, and thus induce sleep in the cat. This constituted the single frequency stimulated group.

To improve the effect, a new pattern of stimulation that combined 2 stimulating frequencies was used to simulate the sleep EEG signal. Firstly, $8 \mathrm{~Hz}$ stimulation was applied to induce a quiet EEG state, then the frequency was reduced to 4 $\mathrm{Hz}$ to further induce the cat to sleep. This group formed the mixed frequency stimulated group.

Each cat was subject to 3 experimental procedures: no stimulation (control), single frequency stimulation and mixed frequency stimulation. Each of these procedures consisted of 8 tests conducted as 4 tests every two days after which the next procedure was applied. The daily testing began at 9:00 $\mathrm{am}$, and the cats were fed with the same food everyday. The stimulating time for each of the two stimulated groups was 20 min. and the times for mixed frequency group were split equally so that the $8 \mathrm{~Hz}$ stimulation was applied for the first $10 \mathrm{~min}$, followed by the $4 \mathrm{~Hz}$ stimulation for the last $10 \mathrm{~min}$.

Results

According to the above experimental design, each cat was exposed to 2 kinds of stimulation as well as no stimulation (control). As such, the results obtained from all four cats are classified in three groups: the control, the single frequency stimulation and the mixed frequency group.

The data was statistically analyzed. The time to sleep (as defined by the first appearance of slow wave sleep) was measured, and the mean value (x) and standard deviation (s) calculated.(Tab.1) Paired t-test statistics were calculated.

Tab. 1 Duration for the sleep (ms)

\begin{tabular}{l|l|l|l}
\hline & $\begin{array}{l}\text { Control } \\
\text { situation }\end{array}$ & $\begin{array}{l}\text { Single } \\
\text { frequency }\end{array}$ & $\begin{array}{l}\text { Mixing } \\
\text { frequency }\end{array}$ \\
\hline No. 1 & $19.33 \pm 4.01$ & $12.04 \pm 2.78^{* *}$ & $7.19 \pm 3.18^{*}$ \\
No. 2 & $18.21 \pm 1.88$ & $9.96 \pm 2.90^{*}$ & $5.01 \pm 1.04^{*}$ \\
No. 3 & $19.27 \pm 1.94$ & $10.65 \pm 1.57^{*}$ & $5.79 \pm 1.24^{*}$ \\
No. 4 & $21.45 \pm 2.31$ & $13.53 \pm 3.11^{*}$ & $9.22 \pm 2.03^{*}$ \\
\hline
\end{tabular}
Paired t-test statistics versus group $1 * \mathrm{P}<0.01 * * \mathrm{P}<0.05$

The experimental results indicate that the mean time to sleep of the stimulated groups was significantly shorter than control group. The duration to fall asleep in the mixed frequency group was shorter than other groups.

\section{Discussion And Conclusion}

The results show that there is a significant correlation between the application of a low frequency pulsed field magnetic field and the length of time to the first appearance of the slow wave sleep pattern in four mature cats. As such, low frequency magnetic stimulation is shown to couple to the brain and affect EEG activity in cats. Further, magnetic stimulation was shown to be a reasonably effective method for inducing sleep, and the effectiveness is dependant on the pattern of the applied stimulation.

This result not only provides a basic understanding, but also a potentially new therapeutic approach for clinical treatment of common diseases such as insomnia. The potential implications of using external magnetic stimulation to modulate the pattern of EEG activity in the cerebral cortex may also go a long way beyond inducing sleep, and further studies on whether activities such as thought and memory can be influenced by external stimulation may be of great benefit.

Further studies are presently being carried out to investigate the effect of the intensity and the direction of the magnetic field on the stimulated EEG activity. Threshold values where the physical effects of stimulation are demonstrated for these and other parameters are also the subject of further investigation.

\section{Reference}

[1] Barker AT, et al. Magnetic Stimulation of the Human Brain and Peripheral Nervous System: an introduction and the Results of an Initial Clinical Evaluation. Neurosurgery. 1987; 20(1): 100-109

[2] Hufnagel A,et al. Activation of the Epileptic Focus by Transcranial Magnetic Stimulation of the Human Brain. Annals of Neurology. 1990; 27(1): 49--60

[3] Similowski T. Cervical Magnetic Stimulation: a New Painless method for Bilateral Phrenic Nerve Stimulation in Conscious Humans. J. Appl. Physiol. 1989; 67(4): 1311--8 